\title{
Anaesthetic management and perioperative complications during deep brain stimulation surgery: Our institutional experience
}

\author{
Renu Bala1,2, Arvind Chaturvedi', Mihir P. Pandia', Parmod K. Bithal²
}

\begin{abstract}
Background: Deep brain stimulation (DBS) surgery is an established therapeutic option for alleviating movement disorders. It represents unique challenges for anaesthesiologists. We retrospectively reviewed the patients, who underwent this surgery at our institution, to study anaesthetic management and perioperative complications. Materials and Methods: After taking approval from the Institutional Ethics Committee, medical, surgical and anaesthesia records of 67 patients who were admitted to undergo DBS surgery during II years period (January $200 \mathrm{I}$ to December $20 \mathrm{II}$ ) were retrieved and reviewed. Sixty-five patients underwent the procedure. Various anaesthetic events and perioperative complications were noted and appropriate statistical analysis was carried out to analyse the data. Results: Electrode placement under monitored anaesthesia care (MAC) was the most commonly used technique ( $86 \%$ of patients). Intra-operative complications occurred in 16 patients (24\%) whereas post-operative complication occurred in 10 patients (I5.4\%). There was one mortality. Though age $>60$ years and American Society of Anesthesiologists status $>I I$ were found to be the risk factors for post-operative complications in the bivariate analysis; they were not significant in multivariate analysis. Conclusions: We report our experience of DBS surgery, which was performed using MAC in majority of patients, though general anaesthesia is also feasible. Further prospective randomised studies comprising large number of patients are warranted to corroborate our finding and to find out the most suitable sedative agent.
\end{abstract}

Key words: Anaesthesia, awake craniotomy, complications, deep brain stimulation surgery, movement disorders, Parkinsonism

\section{INTRODUCTION}

Deep brain stimulation (DBS) surgery is gaining increasing popularity ever since its introduction in 1987

'Department of Anaesthesiology and Critical Care, Pt. B.D. Sharma PGIMS, Rohtak, Haryana, ${ }^{2}$ Department of Neuroanaesthesia, C.N. Center, All India Institute of Medical Sciences, New Delhi, India

Address for correspondence:

Dr. Arvind Chaturvedi, Department of Neuroanaesthesia,

C.N. Center, All India Institute of Medical Sciences,

New Delhi - 110 029, India.

E-mail: c.arvind61@yahoo.co.in

\begin{tabular}{|l|l|}
\hline \multicolumn{2}{|c|}{ Access this article online } \\
\hline Quick Response Code: & Website: \\
\hline & www.jnaccjournal.org \\
\cline { 2 - 2 } & \\
\hline & \\
\hline
\end{tabular}

for treating intractable Parkinson's disease. United States Food and Drug Administration has given approval for its use in Parkinson's disease, dystonia and tremors. However, its indications and applications are expanding and include other disorders such as Alzheimer's disease, obsessive compulsive disorders, chronic pain and epilepsy. The procedure involves two stages; electrode insertion into the target area and battery placement in chest wall. The target sites are deep brain structures such as subthalamic nuclei (STN), globus pallidus internal (GPi) and ventralis intermedius nucleus. STN stimulation is preferred for Parkinson's disease and GPi

This is an open access article distributed under the terms of the Creative Commons Attribution-NonCommercial-ShareAlike 3.0 License, which allows others to remix, tweak, and build upon the work non-commercially, as long as the author is credited and the new creations are licensed under the identical terms.

For reprints contact: reprints@medknow.com

How to cite this article: Bala R, Chaturvedi A, Pandia MP, Bithal PK. Anaesthetic management and perioperative complications during deep brain stimulation surgery: Our institutional experience. $\mathrm{J}$ Neuroanaesthesiol Crit Care 2016;3:119-25. 
for dyskinesia. High-frequency stimulation of target sites modulates their action and hence improves the symptomatology of the patients. However, the exact mechanism of action of DBS surgery is still unknown. ${ }^{[1,2]}$

The procedure is performed with patient awake and the anaesthetic management of patients undergoing DBS surgery is a very challenging and demanding task due to the combination of factors which may be disease-, age- or procedure-related. The anaesthetic goals are (a) to provide good surgical conditions and patient comfort, (b) to facilitate intra-operative monitoring (microelectrode recording [MER] and macrostimulation for target localisation) and (c) to rapidly diagnose and treat complications, which may arise during the procedure. ${ }^{[1]}$

Since the literature on DBS surgery, especially anaesthetic implications, is scarce; we conducted a retrospective review of patients who underwent this procedure at our institute during last 11 years, with the primary objective to describe the anaesthetic management and various complications encountered during the procedure. The secondary objectives were to study the correlation of perioperative complications with patient-related factors or anaesthetic techniques and whether the occurrence of complications was associated with prolonged hospital stay.

\section{MATERIALS AND METHODS}

This retrospective study included all the patients who underwent DBS surgery from January 2001 to December 2011 (11 years).

Medical records were retrieved to review the details of the patients. Anaesthesia records, surgical records and discharge summaries were reviewed. Patient's demographic profile including age, sex, weight, American Society of Anesthesiologists (ASA) physical status were noted. Pre-operative notes were sought to know the indications for DBS surgery, presence of co-morbid conditions and type of anti-parkinsonian medications. Pre-operative investigations such as haemogram, blood biochemistry, chest X-ray, electrocardiograph (ECG) and magnetic resonance imaging were recorded.

Intra-operative data such as the type of anaesthesia administered, level of sedation as per Ramsay Sedation Scale (RSS), methods to secure airway, duration of surgery and anaesthesia, parameters monitored, fluids administered, urine output were noted. Electrode placement and battery placement whether done in single or two sitting were noted. Any complications pertaining to anaesthesia or surgery such as nausea, vomiting, convulsions, haemodynamic changes, respiratory events if any were also noted. Post-operative data were analysed for days of mechanical ventilation and days of hospital stay.

\section{Statistics}

Statistical analysis was done using Statistical Package for Social Sciences (SPSS) Version 20 ( SPSS Inc, Chicago, IL). The categorical data were compared by applying Chi-square test or Fischer's exact test (wherever applicable). Numerical data were compared using Student's $t$-test/ Mann-Whitney U-test. To compare complications during and after the surgery, McNemar test was applied. Univariate and multivariate logistic regression were applied to find out the risk factors for complications at the end of surgery. The $P<0.05$ was taken as significant.

\section{RESULTS}

A total of 67 patients were admitted to undergo DBS surgery during 11 years (January 2001 to December 2011) period. Two patients were excluded; one for non-cooperation during the surgery and the surgeon wanted surgery in awake patient, another because of intractable hypertension despite anti-hypertensive therapy. Out of remaining 65 patients, 63 patients had both electrode and battery placement whereas in two patients, battery placement was deferred. Of these two, one patient had intracranial haematoma for which craniotomy and evacuation of haematoma was done and the second patient developed fever due to dengue viral infection. Both the patients were discharged from the hospital without the second stage of surgery.

Table 1 shows the demographic profile of the patients. Majority of them were of ASA status II. The most common co-morbidity was hypertension, which was seen in 13 patients $(20 \%)$. It was associated with coronary artery disease in five patients and diabetes mellitus in four patients. Hypertension and diabetes mellitus were well controlled with drugs. In most of the patients,

Table 1: Demographic profile and American Society of Anesthesiologists status

\begin{tabular}{lc}
\hline Parameters & Values \\
\hline Age (years) & $50.8 \pm 15.3(13-75)$ \\
Weight $(\mathrm{kg})$ & $58.6 \pm 13.6(30-90)$ \\
Gender $(\%)$ & \\
Male & $40(61.5)$ \\
Female & $25(38.5)$ \\
ASA-status (\%) & \\
I & None \\
II & $47(72.3)$ \\
III & $18(27.7)$ \\
\hline Age and weight expressed as mean \pm SD and range. Gender and ASA \\
status as number of patients and percentage. ASA=American Society of \\
Anesthesiologists, SD=Standard deviation
\end{tabular}


indication for DBS surgery was Parkinson's disease that is shown in Table 2.

The surgery was accomplished as two-staged procedure in 34 patients and single-stage procedure in 31 patients. The average surgical duration for electrode and battery placement was $7 \mathrm{~h}$ and $2 \mathrm{~h}$, respectively. Frame application was done with local anaesthetic infiltration of scalp at pin sites in all patients. In majority of patients, electrode placement was done under monitored anaesthesia care (MAC) with mild $(n=50)$ or sometimes without sedation $(n=6)$. RSS was used for assessing sedation level (mild sedation $=$ score of $2-3$ ). The drugs used for sedation are depicted in Table 3. Midazolam $(0.03 \mathrm{mg} / \mathrm{kg})$ and fentanyl $(1 \mathrm{mcg} / \mathrm{kg})$ were administered intravenously as boluses whereas propofol was administered in the form of infusion in the dose of $25-50 \mathrm{mcg} / \mathrm{kg} / \mathrm{min}$. Dexmedetomidine was administered in the loading dose of $1 \mathrm{mcg} / \mathrm{kg}$ over $10 \mathrm{~min}$ followed by infusion of $0.2-0.4 \mathrm{mcg} / \mathrm{kg} / \mathrm{h}$. General anaesthesia (GA) with endotracheal intubation was administered to nine patients who had severe dystonia. Standard anaesthesia technique comprising fentanyl $(2 \mathrm{mcg} / \mathrm{kg})$, propofol $(1.5-2.5 \mathrm{mg} / \mathrm{kg})$, rocuronium $(0.1 \mathrm{mg} / \mathrm{kg})$ and oxygen $(35 \%)$ and nitrous oxide $(65 \%)$ in isoflurane $(0.8-1$ MAC) was used. Intra-operative monitoring during MAC included heart rate, ECG, non-invasive blood pressure, oxygen saturation, skin temperature (axillary), end tidal $\mathrm{CO}_{2}$ and urine output with Foley's catheter Oxygen was administered through nasal prongs with the facility of $\mathrm{EtCO}_{2}$ monitoring. Battery placement was done under GA with endotracheal intubation or laryngeal mask airway (LMA) in 46 and 17 patients, respectively. Classic LMA was used in 7 patients and ProSeal LMA in 10. Fifty-one patients were extubated in operating room but two of them required reintubation as their level of consciousness deteriorated. A total of 13 patients were shifted with endotracheal tube for elective ventilation and all of them were extubated in Intensive Care Unit within the first two post-operative days. The reasons for elective ventilation were surgeon's request (5 patients), prolonged surgery $\geq 10 \mathrm{~h}$ (4 patients) and poor level of consciousness ( 4 patients).

Intra-operative complications were observed in 16 patients $(24 \%)$, the most common being hypertension (11 patients); only four of these patients were known hypertensive and remaining had no history of hypertension. Hypertension persisted despite adequate pain relief and anxiolysis in 10 patients, hence anti-hypertensive therapy was given (boluses of labetolol $[n=5]$, esmolol $[n=2]$, diltiazem $[n=1]$ and nitroglycerine infusion $[n=2])$. Post-operative complications were encountered in 10 patients (15.4\%) and the most common were intracranial haemorrhage (ICH) and behaviour problems (2 patients each). Various perioperative complications are depicted in Tables 4 and 5. There was one mortality in our study. This patient developed intra-cranial haematoma postoperatively which was

Table 2: Indications for the surgery

\begin{tabular}{lc}
\hline Indication & $\boldsymbol{n}(\mathbf{0})$ \\
\hline Idiopathic Parkinson's disease & $50(76.9)$ \\
Young onset Parkinson's disease & $6(9.2)$ \\
Dystonia & $9(13.8)$ \\
\hline
\end{tabular}

$n=$ Number of patients

Table 3: Intra-operative data

\begin{tabular}{lc}
\hline & $\boldsymbol{n} \mathbf{( \% )}$ \\
\hline Surgery done as (\%) & $31(47.6)$ \\
Single-stage & $34(52.3)$ \\
Two-stage & \\
Electrode placement done with (\%) & $6(9.2)$ \\
MAC without sedation & $50(76.9)$ \\
MAC with sedation & $9(13.8)$ \\
GA & \\
Sedation with (\%) & $3(4.6)$ \\
Midazolam & $2(3.0)$ \\
Fentanyl & $4(6.1)$ \\
Dexmedetomidine & $41(63.0)$ \\
$\quad$ Mixed & \\
\hline$n=$ Number of patients, MAC=Monitored anaesthesia care, GA=General \\
anaesthesia
\end{tabular}

\section{Table 4: Intra-operative complications}

\begin{tabular}{lc}
\hline Complication & $\boldsymbol{n ( \% )}$ \\
\hline Hypertension & $11(16.9)$ \\
Arrhythmias & $1(1.5)$ \\
Bradycardia & $1(1.5)$ \\
Venous air embolism & $1(1.5)$ \\
Tension pneumocephalus & $1(1.5)$ \\
Bronchospasm & $1(1.5)$ \\
\hline$n=$ Number of patients
\end{tabular}

Table 5: Post-operative complications

\begin{tabular}{lcc}
\hline Complication & GA (\%) & MAC (\%) \\
\hline Intra-cranial haemorrhage & $2(3)$ \\
MCA infarct & $1(1.5)$ \\
Focal deficit & $1(1.5)$ & $1(1.5)$ \\
Behavioural problems & $1(1.5)$ \\
Electrolyte imbalance & $1(1.5)$ \\
Others (fever, fall, etc.) & $3(4.5)$ \\
\hline MCA=Middle cerebral artery, GA=General anaesthesia, MAC=Monitored \\
anaesthesia care
\end{tabular}


evacuated but the patient died due to multiple organ failure after 33 days.

The median duration of hospital stay was ten days. Post-operative complications prolonged hospital stay from mean of 10.7 days to 25.7 days and it was statistically significant $(P=0.02)$.

Though there was a positive correlation of pre-operative hypertension with intra-operative hypertension, it was not significant [Table 6]. Further, no association between the intra-operative complications and post-operative complications was found $(P=0.82)$.In bivariate analysis, age $>60$ years and ASA status $>$ II were found to have a positive correlation with post-operative complications [Table 7]. However, in multivariate analysis, they were not statistically significant [Table 8].

\section{DISCUSSION}

The DBS surgery involves two stages. First, the insertion of electrode into the target areas of the brain, which are small and deeply located. Frame-based imaging is used to locate them. Attachment of frame requires tightening of pins into the scalp which is an intense nociceptive stimulus resulting in abrupt increase in blood pressure and heart rate which may be harmful in patients with hypertension and coronary artery disease. ${ }^{[3]}$ Further, these responses may lead to brain oedema, increase in intracranial pressure or $\mathrm{ICH} .{ }^{[4]}$ We attached frame following local anaesthetic in filtration at pin site although supraorbital and greater occipital nerve blocks have also been used. ${ }^{[5]}$

The patient should be awake and cooperative for macrostimulation testing and administration of anaesthetics may interfere with MER also. ${ }^{[1]}$ Majority of our cases were under MAC with minimal (Ramsay sedation score 2 or 3 ) or no sedation. The frequently used drugs for sedation are midazolam, fentanyl, remifentanil, propofol and dexmedetomidine. Propofol has been most widely used either as a bolus or continuous infusion because of its short half-life, ease of titrability of depth of sedation and excellent patient tolerance. ${ }^{[6]}$ However, there are various drawbacks with this drug such as a tendency to abolish tremors, induce sneezing and attenuation of MER ${ }^{[1]}$ In addition, if target control infusion is used, pharmacokinetic behaviour of propofol may be different in patients with Parkinson's disease. ${ }^{[7]}$ It is devoid of analgesic effects for which opioids are added; both can aggravate respiratory depression. We administered bolus fentanyl $(1 \mathrm{mcg} / \mathrm{kg})$ to our patients whenever we used propofol for sedation and did not observe any respiratory event. Dexmedetomidine is a selective alpha- 2 agonist and is creating a niche for itself in neuroanaesthesia practice owing to its unique
Table 6: Association between pre-existing hypertension and intra-operative hypertension

\begin{tabular}{|c|c|c|c|c|}
\hline \multirow[t]{2}{*}{$\begin{array}{l}\text { Pre-existing } \\
\text { hypertension }\end{array}$} & \multicolumn{2}{|c|}{$\begin{array}{c}\text { Intra-operative } \\
\text { hypertension }\end{array}$} & \multirow[t]{2}{*}{ OR } & \multirow[t]{2}{*}{$P$} \\
\hline & Yes & No & & \\
\hline Present & 4 & 10 & 2.5 & 0.23 \\
\hline Absent & 7 & 42 & & \\
\hline
\end{tabular}

OR=Odds ratio

Table 7: Bivariate analysis of various variables with post-operative complications

\begin{tabular}{lcccc}
\hline & Present & Absent & $\boldsymbol{P}$ & OR with CI \\
\hline Age (years) & & & & \\
$<60$ & 4 & 41 & 0.01 & $5.5(1.3-21.8)$ \\
$>60$ & 7 & 13 & & \\
ASA-status & & & & \\
I and II & 5 & 42 & 0.029 & $4.2(1.0-16.1)$ \\
$>$ II & 6 & 12 & & \\
Gender & & & & \\
Male & 7 & 33 & 0.875 & $1.1(0.2-4.2)$ \\
Female & 4 & 21 & & \\
Stage & & & & \\
Single stage & 3 & 28 & 0.137 & $0.3(0.08-1.4)$ \\
Two-stage & 8 & 26 & & \\
Anaesthesia & & & & \\
GA & 1 & 8 & 0.722 & $1.4(0.16-13.4)$ \\
MAC & 10 & 46 & &
\end{tabular}

Airway

management

$\begin{array}{lcccc}\begin{array}{l}\text { Endotracheal } \\ \text { tube }\end{array} & 8 & 38 & 0.225 & 3.5(0.41-30.8) \\ \text { LMA } & 1 & 16 & & \end{array}$

Extubation

$\begin{array}{lcccc}\text { OT } & 7 & 45 & 0.321 & 0.4(0.1-2.1) \\ \text { ICU } & 4 & 9 & & \end{array}$

Intra-operative complication

\begin{tabular}{lllll} 
Yes & 3 & 13 & 0.822 & $1.18(0.27-5.12)$ \\
No & 8 & 41 & & \\
\hline
\end{tabular}

$\mathrm{GA}=$ General anaesthesia, $\mathrm{MAC}=$ Monitored anaesthesia care, $\mathrm{ASA}=$ American Society of Anesthesiologists, LMA=Laryngeal mask airway, ICU=Intensive Care Unit, $\mathrm{Cl}=$ Confidence interval, OR=Odds ratio, OT=Operation theatre

Table 8: Results of multivariate analysis

\begin{tabular}{lccc}
\hline & $\boldsymbol{P}$ & OR & $\mathbf{9 5 \% ~ C I}$ \\
\hline Age & 0.052 & 0.23 & $0.05-1.01$ \\
ASA-status & 0.215 & 2.83 & $0.61-13.10$ \\
\hline
\end{tabular}

ASA=American Society of Anesthesiologists, $\mathrm{Cl}=$ Confidence interval, $\mathrm{OR}=$ Odds ratio 
properties of haemodynamic stability, analgesia, sedation without respiratory depression. There are reports of successful awake craniotomy conducted using this drug. ${ }^{[8,9]}$ Moreover, it has been reported to abolish dyskinesia associated with propofol in a parkinsonian patient during DBS implant. ${ }^{[10]}$ Rozet et al. in a retrospective review reported that during DBS surgery dexmedetomidine sedation did not interfere with electrophysiological monitoring; provided haemodynamic stability and patient comfort. ${ }^{[11]}$ We also had similar results in four patients in whom we used dexmedetomidine infusion for sedation.

GA with tracheal intubation was administered to nine patients who had dystonia because their continuous movements would have caused difficulty for surgeons to locate the target nuclei. Maltête et al. found that general anaesthesia with propofol was feasible for STN electrode placement in patients with Parkinson's disease but residual motor disability and intensity of stimulation was slightly higher in patients under general anaesthesia. ${ }^{[12]}$ However, their results were not reproduced by subsequent studies by Yamada et al. and Lin et al., who observed no difference between awake technique and general anaesthesia in terms of MER and post-operative outcome. ${ }^{[13,14]}$ We also found both techniques acceptable but in the absence of long-term follow-up of patients in our study, it is difficult to comment on the superiority of one technique over the other.

The second stage or battery placement requires tunnelling which is a painful event, hence performed under GA. The surgery is a minor procedure and of short duration favouring the use of LMA. The main concern is the risks of aspiration which is more in patients with Parkinson's disease. ${ }^{[15]}$ The head tilt towards one side is fraught with risks of displacement of LMA. In our study, out of 17 patients in whom LMA was used, displacement was encountered in one patient and it was repositioned and the patient did not have any respiratory sequelae. ProSeal LMA and classic LMA are stable in different head and neck positions per se. ${ }^{[16]}$ We did not encounter any other adverse event. Literature does not have any comparative study between the use of LMA and endotracheal tube in DBS surgery for battery placement. We opine that use of LMA is safe for battery placement.

A wide array of complications can occur during this procedure such as intra-cerebral haematoma, seizures, ischaemic stroke, hypertension, dyskinesia, etc., In our study, intra-operative complications occurred in 16 patients $(24 \%)$ and post-operative in 10 patients $(15.4 \%)$. Intra-operative complications were slightly higher than the reported incidence of intra-operative complications $(9.3-16 \%)$ probably due to learning curve. ${ }^{[17-19]}$
There was 17\% (11 patients) incidence of intra-operative hypertension. Venkatraghavan et al. reported intra-operative hypertension in $4 \%(7 / 172)$ of patients undergoing DBS surgery and they needed treatment for it. In their study, five patients were known hypertensive. ${ }^{[18]}$ In our series, though there was a positive correlation in four patients between pre-existing hypertension and intra-operative hypertension, it was statistically insignificant. Probably, the small sample size of our study was not sufficient to detect a significant difference.

Cardiac rhythm disturbance may result from central cause (such as stimulation of hypothalamus) during the procedure. We observed rhythm perturbations in 10 patients ( 8 had tachycardia, one had supraventricular arrhythmia whereas another had bradycardia). Disturbances from central cause do not need any pharmacological intervention but require cessation of stimulation. Animal studies have shown that stimulation of paraventricular region in hypothalamus can cause haemodynamic disturbances. ${ }^{[20]}$

$\mathrm{ICH}$ is a devastating complication of DBS surgery and can lead to a permanent neurological deficit. It may be the result of inadequate haemostasis or due to intra-operative hypertension. In our series, two patients (3\%) developed $\mathrm{ICH}$, which is in concordance with the reported incidence in DBS surgery $(2-4 \%) .{ }^{[15]}$ While one of them had no history of hypertension, the second patient was a known hypertensive on therapy. None of them had any episode of intra-operative hypertension. The latter patient died because of multiple organ failure. Blood pressure must be controlled optimally (systolic blood pressure $<140 \mathrm{mmHg}$ or within $20 \%$ of patient's usual range) to prevent risks of intra-cerebral haemorrhage. ${ }^{[21]}$ The reported incidence of intra-operative hypertension in literature is $0.6-3.9 \% .{ }^{[18,19]}$ If intra-operative hypertension persists despite ruling out known causative factors (patient's discomfort, anxiety, pain, central cause), anti-hypertensive medications should be administered.

The incidence of intra-operative respiratory complications during DBS surgery has been reported to be 1.6-2.2\% with airway obstruction in $1.1 \%$ of patients. ${ }^{[18,19]}$ The possibility of airway obstruction and difficult airway access should be considered in patients undergoing surgery under MAC with sedation especially with propofol and narcotic combination. Presence of stereotactic frame can make airway management further difficult. We did not find airway obstruction in any patient. However, we encountered bronchospasm in one patient who was undergoing battery placement under GA with LMA in situ. Aspiration was ruled out and probably light plane of anaesthesia was the reason and it did not require any intervention. 
Other infrequent complications during DBS surgery under MAC are venous air embolism (VAE) and tension pneumocephalus. ${ }^{[22-24]}$ We too encountered one case of VAE in our series. Possible predisposing factor apart from semi-sitting posture is hypovolaemia in these patients. Poor oral intake in these patients is a potential reason for causing hypovolaemia. Slow and continuous egress of cerebrospinal fluid from cranial burr holes predisposes them to pneumocephalus. We too observed tension pneumocephalus in one of our patients confirmed with computed tomography scan. The condition resolved with conservative management (oxygen inhalation) after $18 \mathrm{~h}$ without any sequelae.

In bivariate analysis, our study showed that age $>60$ years and ASA >II were the risk factors for the occurrence of post-operative complications, but in multivariate analysis, no independent association between these factors and post-operative complications was detected. Khatib et al. showed age $>64$ years having strong correlation with complications in patients of DBS surgery. ${ }^{[17]}$ The slight difference of age with our study could be because of health status variability between various demography or due to the difference in the experience of neurosurgeons. With increasing age, the ability to withstand long and stressful procedure become limited. The same holds true for ASA status also. While intra-operative complications were transient and self-limiting and had no influence on hospital-stay; however, the post-operative complications prolonged the hospital stay significantly $(P=0.02)$.

\section{CONCLUSION}

The present study demonstrates that DBS surgery can be accomplished under MAC safely and successfully though GA can also be used for the procedure. Although the retrospective nature of data collection and small sample size are the limitations of our study, it provides important addition to the knowledge of anaesthetic technique in patients undergoing DBS surgery. Large prospective studies are warranted to compare various anaesthetic regimens (awake and sedation with different intra-venous agents) on the outcome of surgery for DBS and especially to find out the usefulness of newer drugs such as dexmedetomidine and remifentanil.

\section{Financial support and sponsorship}

Nil.

\section{Conflicts of interest}

There are no conflicts of interest.

\section{REFERENCES}

1. Poon CC, Irwin MG. Anaesthesia for deep brain stimulation and in patients with implanted neurostimulator devices. $\mathrm{Br} \mathrm{J}$
Anaesth 2009;103:152-65.

2. Venkatraghavan L, Luciano M, Manninen P. Review article: Anesthetic management of patients undergoing deep brain stimulator insertion. Anesth Analg 2010;110:1138-45.

3. Bithal PK, Dash HH, Chouhan RS, Mohanty B. Haemodynamic changes in response to skull-pins application-comparison between normotensive and hypertensive patients. Indian J Anaesth 2002;46:381-3.

4. Uyar AS, Yagmurdur H, Fidan Y, Topkaya C, Basar $\mathrm{H}$. Dexmedetomidine attenuates the hemodynamic and neuroendocrinal responses to skull-pin head-holder application during craniotomy. J Neurosurg Anesthesiol 2008;20:174-9.

5. Watson R, Leslie K. Nerve blocks versus subcutaneous infiltration for stereotactic frame placement. Anesth Analg 2001;92:424-7.

6. Deiner S, Hagen J. Parkinson's disease and deep brain stimulator placement. Anesthesiol Clin 2009;27:391-415.

7. Fábregas N, Rapado J, Gambús PL, Valero R, Carrero E, Salvador L, et al. Modeling of the sedative and airway obstruction effects of propofol in patients with Parkinson disease undergoing stereotactic surgery. Anesthesiology 2002;97:1378-86.

8. Bekker AY, Kaufman B, Samir H, Doyle W. The use of dexmedetomidine infusion for awake craniotomy. Anesth Analg 2001;92:1251-3.

9. Mack PF, Perrine K, Kobylarz E, Schwartz TH, Lien CA. Dexmedetomidine and neurocognitive testing in awake craniotomy. J Neurosurg Anesthesiol 2004;16:20-5.

10. Deogaonkar A, Deogaonkar M, Lee JY, Ebrahim Z, Schubert A. Propofol-induced dyskinesias controlled with dexmedetomidine during deep brain stimulation surgery. Anesthesiology 2006;104:1337-9.

11. Rozet I, Muangman S, Vavilala MS, Lee LA, Souter MJ, Domino $\mathrm{KJ}$, et al. Clinical experience with dexmedetomidine for implantation of deep brain stimulators in Parkinson's disease. Anesth Analg 2006;103:1224-8.

12. Maltête D, Navarro S, Welter ML, Roche S, Bonnet AM, Houeto JL, et al. Subthalamic stimulation in Parkinson disease: With or without anesthesia? Arch Neurol 2004;61:390-2.

13. Yamada K, Goto S, Kuratsu J, Matsuzaki K, Tamura T, Nagahiro $\mathrm{S}$, et al. Stereotactic surgery for subthalamic nucleus stimulation under general anesthesia: A retrospective evaluation of Japanese patients with Parkinson's disease. Parkinsonism Relat Disord 2007;13:101-7.

14. Lin SH, Chen TY, Lin SZ, Shyr MH, Chou YC, Hsieh WA, et al. Subthalamic deep brain stimulation after anesthetic inhalation in Parkinson disease: A preliminary study. J Neurosurg 2008;109:238-44.

15. Flexman AM, Talke PO. Parkinson's disease and deep brain stimulator insertion. In: Newfield P, Cottrell JE, editors. Handbook of Neuroanesthesia. $5^{\text {th }}$ ed. Philadelphia: Lippincott Williams \& Wilkins; 2012. p. 325-30.

16. Brimacombe J, Keller C. Stability of the LMA-ProSeal and standard laryngeal mask airway in different head and neck positions: A randomized crossover study. Eur J Anaesthesiol 2003;20:65-9.

17. Khatib R,Ebrahim Z, Rezai A,Cata JP,Boulis NM,JohnDoyle D, et al. Perioperative events during deep brain stimulation: The experience at cleveland clinic. J Neurosurg Anesthesiol 2008;20:36-40.

18. Venkatraghavan L, Manninen P, Mak P, Lukitto K, Hodaie M, Lozano A. Anesthesia for functional neurosurgery: Review of complications. J Neurosurg Anesthesiol 2006;18:64-7.

19. Kenney C, Simpson R, Hunter C, Ondo W, Almaguer M, Davidson A, et al. Short-term and long-term safety of deep brain stimulation in the treatment of movement disorders. J Neurosurg 2007;106:621-5. 
20. Mack SO,Wu M,Kc P,Haxhiu M.Stimulationofthehypothalamic paraventricular nucleus modulates cardiorespiratory responses via oxytocinergic innervation of neurons in pre-Botzinger complex. J Appl Physiol 2007;102:779-83.

21. Gorgulho A, De Salles AA, Frighetto L, Behnke E. Incidence of hemorrhage associated with electrophysiological studies performed using macroelectrodes and microelectrodes in functional neurosurgery. J Neurosurg 2005;102:888-96.

22. Moitra V, Permut TA, Penn RM, Roth S. Venous air embolism in an awake patient undergoing placement of deep brain stimulators. J Neurosurg Anesthesiol 2004;16:321-2.

23. Hooper AK, Okun MS, Foote KD, Haq IU, Fernandez HH, Hegland D, et al. Venous air embolism in deep brain stimulation. Stereotact Funct Neurosurg 2009;87:25-30.

24. Jain V, Prabhakar H, Rath GP, Sharma D. Tension pneumocephalus following deep brain stimulation surgery with bispectral index monitoring. Eur J Anaesthesiol 2007;24:203-4. 